\title{
Review: antioxidant supplementation does not reduce gastrointestinal cancer
}

Bjelakovic G, Nikolova D, Simonetti RG, et al. Antioxidant supplements for prevention of gastrointestinal cancers: a systematic review and meta-analysis. Lancet 2004;364:1219-28.

Bjelakovic G, Nikolova D, Simonetti R, et al. Antioxidant supplements for preventing gastrointestinal cancers. Cochrane Database Syst $\operatorname{Rev} 2004 ;(4): C D 004183$.

$Q$ Do antioxidant supplements reduce the risk of gastrointestinal cancer?

\section{METHODS}

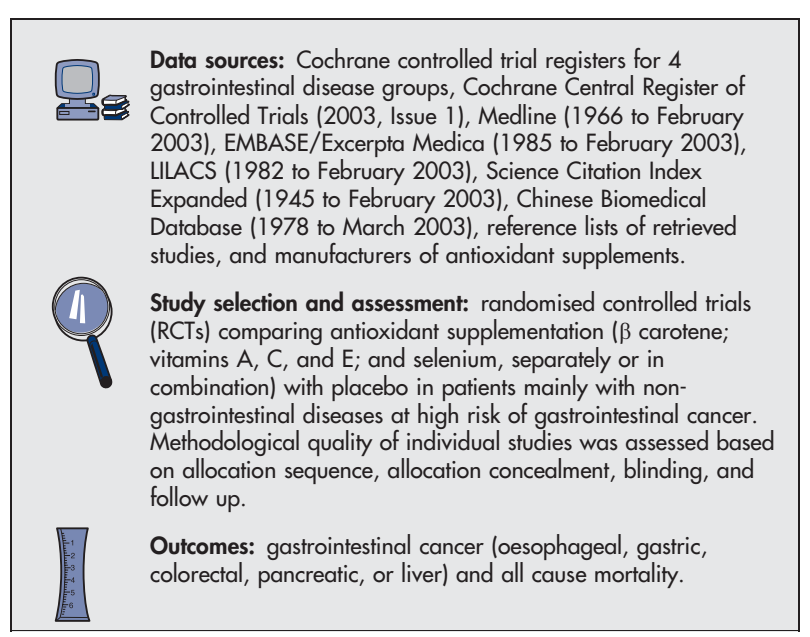

\section{MAIN RESULTS}

14 RCTs ( $\mathrm{n}=170525$, mean age $55 \mathrm{y}$ ) met the selection criteria; 7 had high methodological quality. 13 RCTs provided relevant data on the incidence of gastrointestinal cancer. Antioxidants, regardless of type, did not reduce overall gastrointestinal cancer (relative risk reduction [RRR] $4 \%, 95 \% \mathrm{CI}-4$ to 12 ). This result did not differ for high and low quality trials. In 4 RCTs ( 3 low quality), selenium reduced gastrointestinal cancer more than placebo (RRR 51\%, CI 33 to 64). No other antioxidant or combination reduced gastrointestinal cancer. Meta-analysis of 9 RCTs (2 low quality) using a fixed effects model showed a borderline increase in mortality (relative risk increase [RRI] 5\%, CI 1 to 9), whereas meta-analysis using a random effects model did not (RRI $4 \%, \mathrm{CI}-3$ to 11 ). Analysis of the 7 high quality trials using a fixed effects model showed an increase in mortality with antioxidants (RRI 6\%, CI 2 to 10 ), whereas analysis using a random effects model did not (RRI 6\%, CI -2 to 15 ). Gastroenterology and Hepatology, Medical Faculty, University of Nis, Serbia and Montenegro. goranb@junis.ni.ac.yu

Sources of funding: Knowledge and Research Centre for Alternative Medicine (ViFAB) and Copenhagen Trial Unit.

\section{CONCLUSION}

Antioxidant supplements, with the possible exception of selenium, do not reduce the risk of gastrointestinal cancer and may increase all cause mortality.

A modified version of this abstract appears in ACP Journal Club.

\section{Commentary}

D espite anecdotal evidence to the contrary, a growing body of scientific evidence suggests that antioxidant supplements may not be effective in disease prevention. The meta-analysis by Bielakovic et al adds to that body of evidence, showing no protective effect of antioxidant supplements for the prevention of gastrointestinal cancers in high risk patients.

The most serious and unexpected finding was a higher mortality rate in the antioxidant group than the placebo group. The authors estimate that for every million people taking antioxidant supplements, 9000 premature deaths may have occurred. Possible explanations are that many studies used dosages in excess of current recommended daily intake or that some individuals are inherently more sensitive to antioxidants than others. This finding should be interpreted as preliminary, however, as many of the studies recruited high risk populations. The increased mortality rate therefore may not apply to healthy individuals who take antioxidant supplements as part of a healthy lifestyle.

The results of this review are relevant to public health nurses who work in lifestyle prevention, as well as advanced practice nurses who work in primary care or oncology. Given the increasing numbers of people who take supplements for prevention of disease, the results reinforce the importance of health teaching on the safety and efficacy of unregulated nutritional supplements. In light of these results, it may be prudent to advise high risk patients, such as people who smoke or have high alcohol intakes, of increased risks. However, further studies are needed to determine if the increased mortality rate is, in fact, related to the supplements, which supplements, and at what dosage. Thus, at this time it is difficult to infer harm for all people from supplementation. These findings remind all nurses discussing nutrition with their patients that nutritional supplements should be taken with the same care as regulated, prescribed medications.

Claudia Mariano, RN(EC), MSc East End Community Health Centre Toronto, Ontario, Canada 